\title{
HIGH PREVALENCE OF DISEASE REMISSION IN OLDER PATIENTS WITH TAKAYASU'S ARTERITIS: A BICENTER CROSS-SECTIONAL AND RETROSPECTIVE COHORT STUDY
}

João Calvino Soares de Oliveira ${ }^{1, \star}$, Alexandre Moura dos Santos ${ }^{1}$, Mariana Freitas de Aguiar ${ }^{1}$, Jucier Gonçalves Junior ${ }^{1,}$ Alexandre Wagner Silva de Souza ${ }^{1}$, Rosa Maria Rodrigues Pereira ${ }^{1}$, Samuel Katsuyuki Shinjo ${ }^{1}$

1.Faculdade de Medicina FMUSP, Universidade de São Paulo, São Paulo (SP), Brazil.

*Corresponding author: calvinomed@gmail.com

\section{BACKGROUND}

Takayasu's arteritis (TAK) is a primary systemic vasculitis that affects predominantly young women more often than it affects other groups. Consequently, most available studies that address the status of TAK disease describe young and juvenile patients. No studies have evaluated disease activity comparatively among adults and older patients with TAK, which motivated us to perform the present study.

\section{METHODS}

This bicentric observational, cross-sectional study included 63 consecutive patients with TAK (ACR 1990) undergoing follow-up at two tertiary rheumatology outpatient clinics. The patients were interviewed to collect their current data, as well as data for the preceding 12 months. Patients were divided into four interquartile groups by age and compared in terms of clinical data, laboratory data, disease status, comorbidities (systemic arterial hypertension, diabetes mellitus, dyslipidemia, and fibromyalgia), treatment, and functional status. Disease activity was analyzed using ITAS2010 and NIH scores and acute phase reagents (erythrocyte sedimentation rate and C-reactive protein), whereas disease damage was assessed using the disease extent index (DEI)-Takayasu. Functional status was assessed using the health assessment questionnaire (HAQ).

\section{RESULTS}

The mean age of the patients was $43.0 \pm 10.1$ years; $86.5 \%$ of them were female, and $50.7 \%$ were white. The age groups were divided as follows: Q1 (21-36 years, $n=16$ ), Q2 (37-42 years, $n=18$ ), Q3 (43-49 years, $n=15$ ), and Q4 (50-67 years, $n=18$ ). Disease duration was higher in $Q 4$ compared to other groups $(p=0.027)$. The distribution of comorbidities, vascular impairment (with predominance of Hata $V$ angiographic classification), frequency of patients with disease activity, serum levels of the acute phase reagents, and DEI scores were also comparable among groups. However, in older age, fewer patients used prednisone (Q1: 50.0\%, Q2: 33.3\%, Q3: 6.7\%, Q4: 7.1\%; $p=0.031$,) and immunosuppressive or immunobiological drugs (Q1: 100\%, Q2: 61.1\%, Q3: 46.7\%, Q4: 42.9\%; $p=0.003$ ). Regarding disease activity and damage levels within the preceding 12 months, as well as new TAK-related symptoms or complications, the data were also comparable within the groups, demonstrating no statistical significance.

\section{CONCLUSION}

Takayasu's arteritis older patients appear to require minimal drug intervention, demonstrating more complete disease remission. Even though this reinforces our hypothesis, the results indicate the need for more research focused on the older-TAK population, as scientific studies on this subject remain few.

\section{KEYWORDS}

Takayasu's arteritis, Vasculitis, Older.

\section{REFERENCES}

Arend WP, Michel BA, Bloch DA, Hunder GG, Calabrese LH, Edworthy SM, Fauci AS, Leavitt RY, Lie JT, Lightfoot RW Jr, et al. The American College of Rheumatology 1990 criteria for the classification of Takayasu arteritis. Arthritis Rheum. 1990 Aug;33(8):1129-34. https://doi. org/10.1002/art.1780330811 